\title{
El enfoque comunicativo mediante el tratamiento de la gramática inglesa en la carrera de medicina y estomatología
}

DOI: https://doi.org/10.33262/ap.v2i4.14

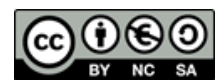

The communicative approach through the treatment of English grammar in the career of medicine and stomatology

Luis Efraín Velastegui López. ${ }^{1}$

\begin{abstract}
.
The continuous development of today's world requires a comprehensive preparation of professionals from all spheres of society, showing mastery not only of a specific specialty in which they work, but also in general and cultural aspects, as well as mastery of a foreign language, especially English, given the reason for being the universal language, since it is not only the mother tongue for many countries, but is spoken as a second language in others.
\end{abstract}

Keywords: continuous development, proficiency, foreign language.

\section{Resumen.}

El continuo desarrollo del mundo de hoy exige de una preparación integral de los profesionales de todas las esferas de la sociedad, mostrando dominio no solo de una especialidad determinada en la cual estos se desempeñen, sino también en aspectos generales y culturales, así como el dominio de un idioma extranjero, especialmente, el inglés, dada la razón de ser el idioma universal, en tanto que no solo es la lengua materna para muchos países, sino que en otros se habla como segunda lengua.

\footnotetext{
${ }^{1}$ Consorcio Ciencia Digital. Ambato, Ecuador. luisefrainvelastegui@cienciadigital.org
} 
Palabras claves: continuo desarrollo, dominio, idioma extranjero.

\section{Introducción.}

El continuo desarrollo del mundo de hoy exige de una preparación integral de los profesionales de todas las esferas de la sociedad, mostrando dominio no solo de una especialidad determinada en la cual estos se desempeñen, sino también en aspectos generales y culturales, así como el dominio de un idioma extranjero, especialmente, el inglés, dada la razón de ser el idioma universal, en tanto que no solo es la lengua materna para muchos países, sino que en otros se habla como segunda lengua.

De ahí la importancia que tiene apropiarse del mismo para intercambiar experiencias entre colegas de diferentes partes del mundo en eventos, congresos, actividades culturales, deportivas y de otra índole; además de favorecer la búsqueda de información en inglés para el desarrollo de investigaciones científicas u otras actividades que lo requieran.

En correspondencia, los programas de estudio de las diferentes carreras universitarias, por una parte, incluyen este idioma como disciplina y por otra, este constituye una estrategia curricular a seguir de conjunto con las demás asignaturas o disciplinas que forman el currículo, en sentido general. De modo que las carreras de la salud no están alejadas de esta situación. Para ello se planifican actividades donde los estudiantes deben mostrar el domino de este idioma, las cuales se concretan en pases de visita, discusiones de casos, entrevistas médico paciente, encuentros de conocimiento en inglés de algunas asignaturas propias de la salud, eventos científicos estudiantiles, entre otras que favorecen la comunicación en este idioma.

La asignatura de Idioma Inglés para la carrera de Medicina, concibe desde el programa el desarrollo de las cuatro habilidades comunicativas, concediéndole prioridad a la habilidad de expresión oral con énfasis en las clases presenciales, así como al desarrollo de las habilidades de lectura, comprensión auditiva y escritura de forma guiada, semi-guiada y productiva.

De esta forma el estudiante deberá ser capaz de comunicarse principalmente de forma oral, sin dejar de desarrollarse en las demás habilidades básicas del idioma. Esto le permitirá un mejor desempeño como futuro profesional, en tanto que gran parte de las actividades realizadas por los mismos (consultas médicas, discusiones de casos, entregas de guardia, pases de visita, entrevistas médico-paciente y médico-médico, entre otras) son indiscutiblemente orales.

Es por ello que las clases de inglés (Clase Práctica Integral de la Lengua Inglesa) en las ciencias médicas están basadas en el enfoque comunicativo, mediante el cual los estudiantes se enfrentan a situaciones lingüístico- comunicativas diversas sin la necesidad de una exposición teórica por parte del profesor, acerca del funcionamiento del lenguaje. Por tanto, 
esto no significa que se dejen de tratar los contenidos gramaticales, por citar un ejemplo, sino hacerlo desde un enfoque comunicativo.

En este sentido el profesor de inglés necesita implementar vías para dar cumplimiento a los principios de este enfoque a través de los contenidos gramaticales, que, a juicio de la autora, no se favorecen desde la enseñanza tradicional de la gramática.

En aras de viabilizar lo anterior es pertinente entonces preguntarnos ¿cómo concebirlo de modo que el estudiante adquiera el conocimiento gramatical?, ¿Qué métodos y medios emplear? y ¿cómo constatar que realmente lo ha adquirido?

Para ello, este trabajo tiene como objetivo vincular las estrategias de aprendizaje, la comunicación educativa, la evaluación educativa y la producción de materiales didácticos y su empleo en las actividades docentes, específicamente en el tratamiento de los contenidos gramaticales del idioma inglés desde un enfoque comunicativo.

\section{Metodología.}

La metodología empleada es de carácter cualitativa. Se utilizó el método analítico-sintético para el estudio de los referentes teóricos-metodológicos y el inductivo-deductivo, para establecer las generalizaciones a partir de los presupuestos teóricos y caracterizar el proceso de enseñanza-aprendizaje del inglés de acuerdo a las estrategias de aprendizaje, la comunicación educativa, la evaluación educativa y la producción de materiales didácticos y su empleo en las actividades docentes, específicamente en el tratamiento de los contenidos gramaticales del idioma inglés desde un enfoque comunicativo.

\section{Discusión.}

El proceso de evaluación de la asignatura inglés en el 3er año de la carrera de medicina. Es necesario señalar qué se ha considerado como Enseñanza Comunicativa de la lengua (Ing. CLT) o Enfoque comunicativo.

Sobre el enfoque comunicativo existe una variedad de investigaciones que han fundamentado su conceptualización teórica-metodológica. Así las diferentes posiciones, han entendido este término como método (Sánchez Pérez, 2005), enseñanza (Richards Jack y Rodgers Theodore, 1986; Melero Abadía, 2005) o enfoque (Brown H. Douglas, 2000). De cualquier modo, más allá de la variedad epistemológica que se asuma, la visión de la enseñanza de lenguas extranjeras es esencialmente comunicativa.

Según Almeida Filho (1993), los métodos comunicativos tienen en común una característica fundamental: el foco en el sentido, en el significado y en la interacción orientada hacia un propósito en la lengua extranjera. La enseñanza comunicativa es aquella que organiza las experiencias de aprendizaje en términos de actividades relevantes y tareas de real interés y/o necesidad del alumno, para que se capacite en el uso de la lengua objeto para realizar acciones 
verdaderas en interacción con otros hablantes/usuarios de esa lengua. Esta enseñanza no toma las formas de la lengua descritas en las gramáticas como el modelo suficiente para organizar las experiencias de aprender otras lenguas, aunque no descarta la posibilidad de crear en el salón de clase, momentos de explicitación de reglas y de práctica rutinizante de los subsistemas gramaticales.

Yalden (1987) resume la esencia de (CLT), de esta forma: "Se basa en la noción de los estudiantes como comunicadores, naturalmente dotado de la habilidad para aprender idiomas. Este persigue proporcionar al estudiante el sistema del lenguaje en cuestión. Se asume que los estudiantes tendrán que prepararse para usar el mismo (de forma oral o escrita) en varios actos de comunicación predecibles e impredecibles que surgen tanto en la interacción en el aula como en situaciones reales, ya sea durante la práctica del idioma o posterior a ella".

González Cancio (2008) sostiene como enfoque comunicativo el criterio planteado por Nunan D. y citado por Font S. en su tesis de doctorado donde expresa: si se asume la definición hecha por Anthony E, en 1963 y citada por Brown D, en 1994: "Un sistema de concepciones relacionadas con la naturaleza del lenguaje, así como la naturaleza de su aprendizaje y de su enseñanza, es posible comprender por qué se habla de enfoque comunicativo. Este tiene que ver con una concepción educativa que está presente en todo lo que ocurre en el aula, y se distingue por nuestras concepciones en lo relacionado a la naturaleza del lenguaje y del aprendizaje".

Según Jack C. Richards, "CLT puede entenderse como un sistema de principios sobre las metas u objetivos de la enseñanza de idioma, cómo los estudiantes aprenden un idioma, los tipos de actividades docentes que facilitan el aprendizaje, y el rol de los profesores y estudiantes en el aula."

En su artículo este afirma que la meta u objetivo del CLT es la enseñanza de la competencia comunicativa, la cual se refiere a la capacidad de usar el lenguaje para la comunicación significativa, es decir, para expresar significados.

Sobre la cuestión de cómo los estudiantes aprenden el idioma, el autor se refiere a una perspectiva vista como resultado de procesos como:

- Interacción entre el estudiante y los usuarios del idioma.

- Creación colaborativa del significado

- Creación de interacciones con un propósito y un significado a través del lenguaje.

- Negociación del significado de modo que el estudiante y su interlocutor logren comprenderse.

- El aprendizaje atendiendo a la retroalimentación que adquieren los estudiantes al hacer uso del idioma.

- Prestar atención al lenguaje que se escucha (input) y tratar de incorporar nuevas formas al suyo propio, desarrollando la competencia comunicativa. 
- Tratar y experimentar diferentes formas de decir las cosas.

El tercer elemento se refiere a las actividades de trabajo en parejas, en grupos, juego de roles y trabajos prácticos.

En relación al rol del profesor y los estudiantes, se espera que los estudiantes tengan un mayor grado de responsabilidad por su propio aprendizaje, y los profesores asuman el rol de facilitador y monitor.

La autora de este trabajo considera que la posición de Cancio no explica claramente los rasgos esenciales que nos permitan identificar el enfoque comunicativo, en tanto que el hecho de que este enfoque tiene que ver con una concepción educativa que está presente en todo lo que ocurre en el aula, resulta ambiguo. Por lo tanto, es necesario particularizar más en sus rasgos distintivos.

En cambio, los demás autores también hablan de la naturaleza del lenguaje, pero ofrecen más detalles en lo relacionado la interacción en el aula, las situaciones de la vida real, así como el rol de los estudiantes y los profesores. No obstante, en este trabajo se asume la definición de Jack C. Richards por considerarse más completa.

Independientemente de las diferentes definiciones, muchos de estos autores coinciden en que la esencia del enfoque comunicativo es la competencia comunicativa. Como todo enfoque o concepción, el enfoque comunicativo se caracteriza por una serie de principios que organizan el proceso como un todo. En correspondencia con esto, diferentes investigadores, quienes han profundizado en el enfoque comunicativo, han hecho referencia a una gran variedad de principios, que difieren en número entre tres, cinco y ocho, etc.

Sin embargo, a pesar de esa situación es válido apuntar que en sentido general todas esas posiciones concuerdan en su significación y funciones, por ejemplo:

Berns (1990) brinda un resumen de ocho principios para el CLT:

1. La enseñanza de lenguas está basada en una visión del lenguaje como comunicación. O sea, el lenguaje es visto como una herramienta social que usan los hablantes para construir significado; los hablantes comunican algo a alguien por algún propósito, ya sea oralmente o de forma escrita.

2. La diversidad es reconocida y aceptada como parte del desarrollo y uso del lenguaje en estudiantes y usuarios del mismo como segunda lengua, del mismo modo que en los usuarios de este como lengua materna o primera lengua.

3. La competencia de un estudiante se considera en términos relativos, no absolutos.

4. Se reconoce más de una variedad de un idioma como modelo viable para el aprendizaje y la enseñanza. 
5. Se reconoce la cultura como un instrumental en la formación de la competencia comunicativa del hablante, tanto en su lengua materna como en su segunda lengua.

6. No se prescribe una sola metodología o conjunto de técnicas fijas o específicas.

7. Se reconoce que el uso del lenguaje realiza una función idealista, interpersonal y textual, y está relacionado con el desarrollo de la competencia del estudiante en cada una de ellas.

8. Es esencial que los estudiantes realicen cosas con el lenguaje - o sea, que usen el lenguaje con varios propósitos en todas las fases del aprendizaje.

Neil Naiman (1989).

\section{La práctica significativa más allá del nivel oracional}

Este principio nos enseña que el lenguaje opera a niveles superiores a palabras u oraciones aisladas, que el mismo adquiere significado en el contexto; que la práctica en el aula debe propiciar que los estudiantes experimenten con lo que conozcan del idioma para alcanzar propósitos comunicativos reales (oralmente o de forma escrita); y procesar partes del lenguaje como estos ocurren en la vida real (escuchar y leer). Este principio también tiene implicaciones en términos de la motivación del estudiante, en tanto que implica pedirles que comuniquen y procesen significados reales del lenguaje.

\section{La clase centrada en el estudiante.}

Este principio plantea que si se espera que los estudiantes desarrollen habilidades, entonces se les deben dar varias oportunidades de participar en parejas, en grupos, y para toda el aula. En otras palabras, este principio implica que los profesores deben propiciar el momento para que los estudiantes actúen.

3. Orientación de tareas para actividades en el aula.

Este principio está relacionado a la enseñanza problémica. Esta plantea que los estudiantes deben aprender mediante la solución de tareas y problemas cercanos a la vida real. En la vida real uno no busca una noticia con el propósito de buscarla, sino más bien para darle seguimiento a un suceso actual.

4. Desarrollo de estrategias de aprendizaje fuera del aula

Este principio reconoce la necesidad de que los estudiantes desarrollen habilidades y formas de aprendizaje a su propio modo. En otras palabras, ellos no solo deben adquirir conocimientos y hábitos, sino también desarrollar habilidades, en tanto que es imposible enseñarle a alguien, todo lo que necesita saber; mas bien, se les debe ayudar a ser capaces de aprender a por si mismos. 


\section{Corrección en pareja y trabajo en grupo}

Este principio considera que para que los profesores ayuden a desarrollar la exactitud en los estudiantes, estos deben emplear técnicas de corrección por retroalimentación. Mediante el uso de estas técnicas, los estudiantes desarrollan habilidades de autocorrección y autoevaluación. Obviamente, si los estudiantes son capaces de corregir sus propios errores, seguramente tendrán la habilidad de corregir el de sus compañeros del mismo modo, mientras trabajan en grupos o en parejas, e incluso en situaciones fuera del aula.

Richards y Rogers plantean los siguientes:

- Principio de la comunicación: las actividades que implican la comunicación real, promueven el aprendizaje.

- Principio de tareas: las actividades en las que el lenguaje es usado para realizar tareas significativas, promueven el aprendizaje.

- Principio de significación: el lenguaje que es significativo para el estudiante, promueve el proceso de enseñanza-aprendizaje.

Teniendo en cuenta todo lo anterior expuesto y lo indicado en el artículo 33 de los Guiones Metodológicos Generales, en relación a que la Clase Práctica Integral de la Lengua Inglesa (CPILI) en las ciencias médicas se basa en el enfoque comunicativo; supone entonces el cumplimiento de los principios del mismo, cuestión esta que se torna compleja en la enseñanza de los contenidos gramaticales, específicamente los que muestra el programa de Inglés para tercer año de medicina y estomatología, en tanto que los ejercicios y/o actividades propuestos en el cuaderno de trabajo, prestan mayor atención al uso de las formas o estructuras gramaticales de manera aislada, que a la comunicación de ideas donde se emplee la gramática en un contexto.

En este trabajo se muestran dos actividades que a juicio de la autora pueden ser útiles para el desarrollo de otras por parte de los docentes que enfrentan esta situación y que a su vez permiten implementar el enfoque comunicativo mediante sus principios al tiempo que se imparte un contenido gramatical.

Las referidas actividades que se muestran como ejemplos son las siguientes:

Actividad \# 1: Es un trabajo en pareja (pair work activity).

Primeramente, el profesor explica en qué consiste la actividad para evitar interrupciones cuando la misma esté en progreso. Si los estudiantes están seguros, entonces se les pedirá organizarse en parejas. Luego, a cada pareja se le dará una situación que deberán dramatizar.

Situaciones. (Role plays): 
1. A: You are feeling nervous lately. This situation appears whenever you have a test, so you decided to see a psychologist. Talk about your situation in order to find a solution. Tell him/her when you feel like that.

1. B: You are a psychologist. A patient comes to you because he/she is feeling nervous lately. Ask him/her when he/she feels like that in order to find a solution. Give $\mathrm{him} /$ her a piece of advice.

2. A: You notice that your friend looks stressed. Comment that to him/her and ask if he/she feels like that very often. Give him/her some advice.

2. B: Your friend notices that you look stressed. It frequently happens when you are too busy at work. Answer your friend's question and ask for some advice.

3. A: You and your friend are studying for the Pharmacology test in the afternoon. $\mathrm{He} / \mathrm{she}$ looks really tired, so you say that to him/her. Ask for information to know when he/she feels that way. Give advice.

3. B: You and your friend are studying for the Pharmacology test in the afternoon. $\mathrm{He} /$ she notices you are really tired. It always happens when you stay up late at nights. Answer your friend's questions.

4. A: You feel sad whenever you are far from your family. Now you are fulfilling an internationalist mission. Tell one of your colleagues how you feel. Answer your colleague's question. Ask how he/she feels, too.

4. B: You are fulfilling an internationalist mission with some other colleagues. One of them is sad. Ask about his/her problem. Say how you feel too. Give psychological support.

5. A: You have just received some bad news and you feel depressed. You usually feel like that after this kind of situation. Talk about that with your best friend.

5. B: Your friend looks depressed. Ask about his/her problem. Try to find out if he/she only gets depressed after he/she receives bad news. Give a logical end to the conversation.

Aunque estas son situaciones imaginarias, ellas pueden encontrarse en el mundo real. Por lo tanto, los estudiantes se encuentran inmersos en una actividad en la que cada pareja puede interactuar uno con el otro, empleando funciones comunicativas que permitan el uso funcional del contenido gramatical. Particularmente, en este caso estamos trabajando con oraciones adverbiales de tiempo, las cuales expresan cuándo se realiza una acción. De esta forma, expresiones como: (Yo siempre me deprimo.../ me canso.../ me estreso..., etc., cuando.../ cada vez que.../ antes de.../ después que...) lejos de ser usadas de forma aislada, 
estarán acompañadas de otras funciones comunicativas que conforman la conversación dentro de un contexto, forma tal que la situación parezca más real. Además, las cuatro habilidades del idioma se ponen en práctica, aunque escuchar y hablar son las más usadas en este caso.

Al finalizar la actividad el profesor dejará de pasar por los puestos, tomando notas sobre el desempeño de los estudiantes. Le pedirá a una o dos parejas que dramaticen su situación en frente del aula, mientras el resto prestará atención. Concluido esto, se chequean algunos errores generales y se prepara a los estudiantes para la próxima actividad.

Actividad \# 2: Trabajo en grupo (Group work activity).

\section{Encuentra a alguien que...(Find someone who...)}

- Likes dermatology/ cardiology/ orthopedics/gynecology...

- Is assisting a patient with a chronic disease.

- Loves children.

- Has been affected by his/her patients' problems.

- Has performed a surgery.

- Has assisted a hypertensive patient.

- Is planning to travel for collaboration.

- Has had a patient with pneumonia.

- Enjoys working with elderly people.

Eg.1: Do you...? / Are you...? / Have you ...?

a) Then report to the class through an adjective clause. Eg.2: $\underline{\mathbf{X}}$ is a girl/boy who...

Para el desarrollo de esta actividad en grupo, el profesor divide el aula en grupos pequeños (5-6 estudiantes) de acuerdo al número de estudiantes con que se cuenta en el aula. También es necesario aclarar bien el primer ejemplo, ya que no todos los incisos están enfocados en tiempo presente simple con verbos de acción, sino que además hay otros con la forma del verbo to be, así como el tiempo presente prefecto. Esta explicación es de vital importancia para que los estudiantes logren formular las preguntas de $\mathrm{Si} / \mathrm{No}$ (Yes/ No questions) necesarias para obtener información de manera más fácil. Se puede notar claramente, como los estudiantes necesitan encontrar qué compañero guarda relación con estas situaciones o no, de modo que puedan reportar la información al resto del aula por medio de las oraciones 
subordinadas adjetivas, como en el segundo ejemplo de la actividad: Jenny is a girl who... (Jenny es una chica que...), y así sucesivamente, hasta que la actividad se complete.

En otras palabras, existe un vacío de información, cuya respuesta la tiene el compañero al cual se le debe preguntar, por lo tanto, hay una razón para hablar y hacer preguntas, es decir, existe una necesidad de comunicarse; lo cual hace que la conversación no sea forzada. Por otra parte, todos los incisos están contextualizados y diseñados en correspondencia con la experiencia personal de los estudiantes. En fin, esta actividad está dirigida a reafirmar la formación de los estudiantes de acuerdo a sus necesidades profesionales e intereses como futuros médicos.

En otro orden de cosas hay que tomar en cuenta que el centro del proceso de enseñanzaaprendizaje es el estudiante, por lo que la dirección del mismo debe pensarse desde la implementación de las estrategias de aprendizaje “...con las cuales los estudiantes [...] aprendan a ser responsables de su aprendizaje en la medida en que ganen capacidad de autogestión y autonomía en la toma de decisiones sobre cómo orientar su aprendizaje fuera del aula (Usma y Frodden, 2003; y citado por Luz Mery Orrego y Ana Elsy Díaz, 2010).

En el caso de las actividades mostradas, aunque están concebidas para desarrollarse en el aula, se ponen de manifiesto algunas de las estrategias ofrecidas por Guillermo Bernaza como son: las estrategias generales de adquisición de conocimiento, específicamente las de reproducción y elaboración; además de las estrategias de autoevaluación y autorregulación, así como estrategias para aprovechar no solo el apoyo del profesor, sino también de sus compañeros de estudio.

No obstante, muchas son las alternativas que en el caso de las ciencias médicas se pueden aprovechar para darle salida a las estrategias de aprendizaje en la asignatura de inglés, como es el desarrollo y empleo de materiales digitales en la práctica docente, entre los que se pueden mencionar, presentaciones, diccionarios y glosarios bilingües digitales con imágenes y sonidos, páginas webs, entre otros que, gracias a la introducción de las tecnologías de la información y la comunicación (TICs), permiten el desarrollo de las competencias comunicativas en los estudiantes, y con ello el perfeccionamiento del proceso enseñanzaaprendizaje de la asignatura.

Todo este proceso pasa indudablemente por el sistema de evaluación de la asignatura Inglés $\mathrm{V}$ y que viene estipulado desde el programa. Para ello... "Se realizaran evaluaciones frecuentes, dos Trabajos de Control en Clase (oral y escrito) y un Trabajo de Control Extraclase (TCE) y examen final. Las frecuentes se realizarán en clase a través de: conversaciones, preguntas y respuestas, dramatizaciones, narraciones, descripciones, monólogos, etc. 
La evaluación frecuente, al apoyarse en el desempeño del estudiante durante la actividad docente, resulta la de mayor significación en el proceso de aprendizaje. Será utilizada para valorar sistemáticamente la efectividad de la autopreparación de los estudiantes.

Se puede observar que persiste la tendencia reduccionista de la evaluación, atendiendo a la aplicación de exámenes y evaluaciones cuantitativas en el PEA del Inglés V, lo cual influye negativamente en el estudiante en cuanto a la motivación, el interés, la autoestima; aunque se ha concedido importancia a la evaluación cualitativa apoyada en el desempeño del estudiante como una vía para obtener información que sirva de base al docente para el perfeccionamiento de este proceso.

Siendo así, le corresponde al docente emplear las formas de evaluación más apropiadas, mediante la retroalimentación, la reflexión, la observación, así como promover la autoevaluación en los estudiantes, sin abandonar las funciones de diagnóstico, instructiva, educativa, de desarrollo y de control que debe cumplir la misma, según especialistas del Ministerio de Educación y reafirmado por Luis Manuel Gaínza Lastre en su artículo “Aprendizaje y evaluación del idioma inglés en las escuelas de idioma”.

\section{Conclusiones.}

- El Enfoque Comunicativo como forma esencial de enseñanza en la CPLI para la carrera de medicina permite el desarrollo de las habilidades comunicativas en los estudiantes, a la vez que interactúan entre ellos de forma oral, y contextualizada, sin dejar de desarrollar las cuatro habilidades básicas del idioma. Esto le facilitará un mejor desempeño como futuro profesional al enfrentar actividades propias de su profesión (consultas médicas, discusiones de casos, entregas de guardia, pases de visita, entrevistas médico-paciente y médico-médico, entre otras) en países de habla inglesa, como parte de la colaboración médica.

- Existen limitaciones en el cuaderno de trabajo Vision III de la asignatura de inglés V en cuanto al diseño de las actividades o ejercicios propuestos para dar tratamiento a la gramática desde este enfoque comunicativo.

- La muestra de actividades ofrecida en este trabajo, a juicio de la autora, contribuye a mejorar esta situación y sirve de base para la elaboración de otras por parte de los profesores de la asignatura en cuestión.

- La evaluación de la asignatura Inglés V en las carreras de medicina y estomatología debe asumir además de lo cuantitativo, los aspectos valorativos de la cualidad del desempeño de los estudiantes y que reflejen el dominio de los contenidos, el desarrollo de habilidades y sus competencias para comunicarse en este idioma, de modo que contribuya de mejor manera a alcanzar resultados satisfactorios en la motivación e interés por su estudio, así como en el proceso formativo de los futuros egresados de las carreras de las ciencias médicas. 


\section{Referencias bibliográficas.}

Alice C. O`Maggio, Heinle. Teaching Language in Context: proficiency-oriented instruction. Heinle Publishers, Boston, 1986, pp. 7-8.

Bermello-Lastra, Gladys y Vega-Puente, Juan Carlos. An English Grammar for Spanish Speaking Teachers of English. Universidad Pedagógica Enrique José Varona. Departamento de idiomas extranjeros. La Habana. 2005

Bernaza Rodríguez, Guillermo. Algunas estrategias de aprendizaje para estudiantes de las SUM. Material digitalizado, (s/f).

Colectivo de autores. Vision III. Student's book. Ciudad de La Habana: Ed. Ciencias Médicas. 2009.

Deckert, Glenn. The Communicative Approach: Adressing Frequent Failure. Tomado de la revista “English Teaching Forum”. Enero 2004, Vol. 42, No 1, Páginas 12-13.

Gabbiani, Beatriz. Enseñar lengua: reflexiones en torno al enfoque comunicativo. Revista Quehacer Educativo, Diciembre, 2007.

Gaínza Lastre, Luis Manuel 2012. Aprendizaje y evaluación del idioma inglés en las escuelas de idioma. Revista Transformación, ISSN: 2077-2955, enero-julio 2012, 8 (1), 74-84.

González Cancio, Roberto. La clase de lengua extranjera. VARONA, Revista CientíficoMetodológica, No. 46, pp.52-59, 2008.

Neil Naiman. "Teaching Pronunciation Communicatively". Published in Communication Journal, INTUR, 1989.

Orrego, Luz Mery; Díaz Monsalve, Ana Elsy: Empleo de estrategias de aprendizaje de lenguas extranjeras: inglés y francés. Íkala, Revista de Lenguaje y Cultura. Vol. 15, No. 24, enero-abril 2010.

Programa de la asignatura Inglés V y Vl de las carreras de medicina y estomatología. 2010.

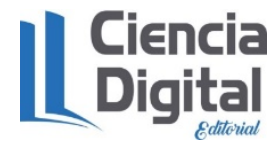




\section{Para citar el artículo indexado}

Velasteguí López, L. E. (2020). El enfoque comunicativo mediante el tratamiento de la gramática inglesa en la carrera de medicina y estomatología. AlfaPublicaciones, 2(4), 25-37. https://doi.org/10.33262/ap.v2i4.14

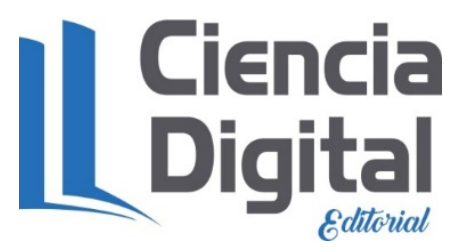

El artículo que se publica es de exclusiva responsabilidad de los autores y no necesariamente reflejan el pensamiento de la Revista Alpha Publicaciones.

El artículo queda en propiedad de la revista y, por tanto, su publicación parcial y/o total en otro medio tiene que ser autorizado por el director de la Revista Alpha Publicaciones.
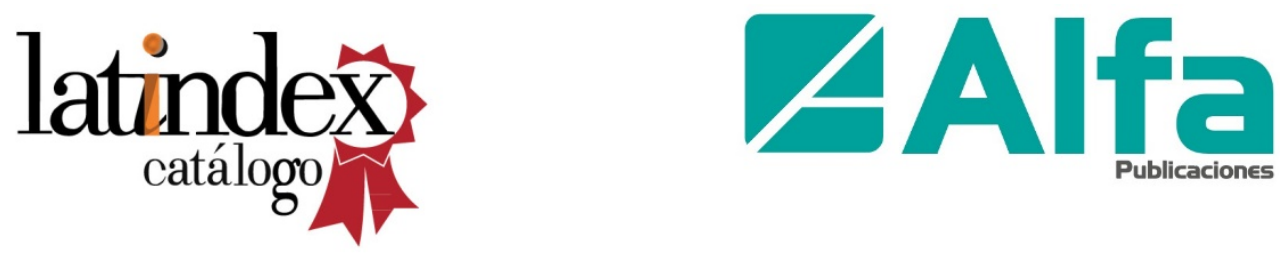